\title{
Quasi-Hexagonal Vortex-Pinning Lattice Using Anodized Aluminum Oxide Nanotemplates**
}

\author{
Xavier Hallet, Mária Mátéfi-Tempfli, Sébastien Michotte, Luc Piraux, \\ Johan Vanacken, Victor V. Moshchalkov, and Stefan Mátéfi-Tempfli*
}

The interest in the template-based synthesis of nanomaterials and nanostructures is continuously increasing due to the advantageous features offered by the templates and the ease of processing a large number of nanostructures in parallel. In recent years, nanoporous anodic aluminum oxide (AAO) has been intensively studied and used for that purpose. ${ }^{[1]}$ At present, it is considered one of the most promising templates $^{[2]}$ mainly because of its unique features, which include easily tunable geometrical parameters, mild preparation conditions, and resistance to high temperatures. AAO, which is a typical self-ordered nanopore-array material formed by the electrochemical oxidation of $\mathrm{Al}$ in acidic solutions, is an excellent starting host material for the fabrication of various nanomaterials and nanostructures. It possesses a hexagonally packed arrangement of nanoscale-sized straight blind holes aligned perpendicular to the surface. The voids of the nanoscale-channeled structure are typically filled with various materials and heterostructures using electrochemical or chemical vapor deposition methods after the elimination of the oxide barrier layer that closes the bottom ends of the holes. While this barrier layer is typically considered undesirable and eliminated, herein we emphasize that the surface of this layer possesses an ordered array of hemispheres that can also be used as a very interesting template. We describe the advantages of the ordered bumpy surface for creating artificial superconducting pinning centers in $\mathrm{Nb}$ films deposited on it.

[*] Dr. S. Mátéfi-Tempfli, X. Hallet, M. Mátéfi-Tempfli, Dr. S. Michotte, Prof. L. Piraux

Physico-chimie et physique des matériaux

Université catholique de Louvain (UCL)

Place Croix du Sud 1, Louvain-la-Neuve, B-1348 (Belgium)

E-mail: matefi@uclouvain.be

Prof. J. Vanacken, Prof. V. V. Moshchalkov

Institute for Nanoscale Physics and Chemistry

Katholieke Universiteit Leuven (KUL)

Celestijnenlaan 200D, 3001 Leuven (Belgium)

[**] This work was supported by the Interuniversity Attraction Pole Program (P6/42), Belgian State, Belgian Science Policy. X. H. acknowledges financial support from the Fund for Training in Research in Industry and Agriculture. S. M. is a post-doctoral researcher for the National Fund for Scientific Research. The authors would like to thank Bas Opperdoes for the MBE growth of the $\mathrm{Nb}$ thin films.

DOI: $10.1002 /$ smll.200900400
In type II superconductors, the penetration of quantized magnetic flux bundles, called vortices, allows superconductivity to be maintained at high magnetic fields. Moreover, Abrikosov showed that vortices tend to form a regular lattice. ${ }^{[3]}$ Nevertheless, an electrical current exerts a force on the vortices that can set them in motion and creates a dissipative state. Vortex pinning has thus attracted much attention for decades due to technological and scientific interest. Lithography allows achievement of and control over a tremendous variety of patterns of pinning centers. ${ }^{[4,5]}$ The interaction of various pinning site lattices with the Abrikosov lattice has shown very interesting behaviors such as the so-called matching effect. ${ }^{[4,5]}$ However, lithographic techniques are expensive and time-consuming, which limits their practical applicability. Recently, self-assembled techniques have become a very promising way to increase the density of pinning centers at low cost. Previously, Welp et al. used AAO templates to create a triangular lattice of antidots, which resulted in an increase by two orders of magnitude of the lithography-based matching field best achieved so far. ${ }^{[6]}$ Vinckx et al. also used self-assembled polystyrene nanospheres to create a hexagonal lattice of pinning centers. $^{[7]}$

In our previous studies we have considered the effects of pore size, pore spacing, and film thickness on the matching fields and vortex pinning. ${ }^{[7-9]}$ In this work, we present a new approach to create an interesting type of pinning lattice with the AAO template by using the barrier layer face of the AAO (bottom surface, Figure 1) to modulate the thickness of thin $\mathrm{Nb}$ films (a)
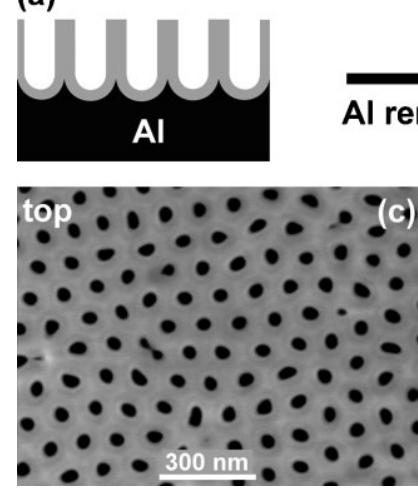

Al removal (b)

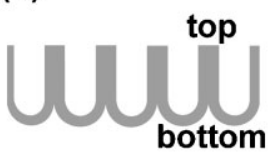

Figure 1. a) Schematic representation of the AAO template after the twostep anodization process and b) after the aluminum removal. Scanning electron microscopy (SEM) pictures of both surfaces of the obtained template: c) top and d) bottom. 
evaporated on it. We show that this surface can be used for efficient vortex pinning as well as the creation of a quasihexagonal lattice of pinning centers. We compare the results obtained with this approach with a sample containing a lattice of antidots (realized on the top surface, Figure 1). We show that the major advantages of the barrier layer face are that it can pin twice as many vortices and that matching effects are maintained at higher fields and lower temperatures.

Self-ordered nanoporous alumina templates with 100-nm pore spacing were prepared in order to create the two different patterns in $\mathrm{Nb}$ thin films. The AAO templates were fabricated by a two-step anodization process in order to obtain highly ordered structures. The pores consist of long channels closed at the bottom end by a round-shaped $\mathrm{Al}_{2} \mathrm{O}_{3}$ barrier layer (Figure 1a and b). The top surface (Figure 1c) contains a triangular lattice of holes, which has already been employed for various purposes such as imprinting triangular lattices of antidots in thin superconducting films. ${ }^{[6,9]}$

In the present work, we introduce the use of the barrierlayer surface (at the bottom) composed of a triangular lattice of hemispheric nanoscale bumps that possess the same spacing as the top layer $(100 \mathrm{~nm})$, but with a completely different surface morphology. Figure 1d illustrates this barrier-layer surface that shows the triangular arrangement of bumps with an average domain size for the highly ordered regions of about $1 \mu \mathrm{m} .^{[10]}$ The $\mathrm{Nb}$ layers were deposited using molecular beam epitaxy (MBE) and different $\mathrm{Nb}$ thicknesses and angles of evaporation were studied. The paper presents the most striking results obtained on the bumpy surface, which are those obtained for a 50 -nm-thick $\mathrm{Nb}$ film evaporated under $30^{\circ}$ from normal. At this deposition condition an asymmetric thickness modulation is induced, creating two interpenetrating triangular lattices of pinning centers. These results are compared to those obtained on a triangular lattice of antidots realized by using a $50-\mathrm{nm}$ thick $\mathrm{Nb}$ film evaporated perpendicularly to the top AAO surface.

The barrier-layer surface (at the bottom) looks like a triangular lattice of domed hexagons (Figure 1d). The middle point of each bump (dome) is a local maximum and the hexagon corners are local minima (interstices). As the evaporation is performed under an angle, the bumps allow shadowing effects. In fact, depending on the evaporation direction, small areas close to the interstices will have a smaller thickness. This thickness modulation creates pinning centers located nearby each interstice. As there are two times more interstitial sites than bumps, this technique allows two times more pinning centers for a given template using its bottom face (barrier layer) rather than its top face (holes). Nevertheless, the thickness modulation strongly depends on the local orientation of the bump lattice compared to the direction of evaporation. Indeed, if the evaporation direction corresponds to a row of bumps, the thickness modulation will be the same for every interstice. However, if the evaporation direction corresponds to a hexagon side, the thickness modulation will not be equivalent for each interstice. Half of the interstitial sites will then undergo a strong shadowing effect (strongly reduced thickness) while the other half will undergo a smaller shadowing effect (lightly reduced thickness). In this case, the overall thickness modulation will create two interpenetrating triangular-pinning-center lattices with different strengths. As the sample is not perfectly ordered but is composed of several domains having different orientations, it is not possible to match the evaporation direction with every particular lattice direction. Therefore, the mean pinning lattice will be a mix of the two cases presented above, leading to two interpenetrating triangular lattices with different strengths equivalent to a quasi-hexagonal lattice of pinning centers.

The matching field for a triangular lattice of pinning centers (holes) can be obtained with

$H_{1}^{\mathrm{top}}=\frac{2 \Phi_{0}}{\sqrt{3} \cdot d^{2}}$

where $d$ is the pinning-center spacing and $\Phi_{0}$ is the magnetic flux quantum $\left(2 \times 10^{-15} \mathrm{~Wb}\right)$. This leads to $H_{1}^{\text {top }}=215 \mathrm{mT}$ $(d \approx 100 \mathrm{~nm})$ in our case. For the quasi-hexagonal lattice of pinning sites (near the interstices), the matching field can be easily obtained from Equation (1), remembering that the total number of pinning centers has been doubled. This means that the matching field in this case is also doubled:

$H_{1}^{\text {bottom }}=2 \cdot H_{1}^{\text {top }}$

Figure 2a shows the critical-current curves of the $\mathrm{Nb}$ film with a triangular antidot lattice (deposited on the top face of the template). When each hole is filled with one fluxon (Figure 3a) at the first matching field $\left(H_{1}^{\text {top }}=215 \mathrm{mT}\right)$, a pronounced increase of the critical current is observed as already reported. ${ }^{[9,11]}$ After the first matching field, the critical current
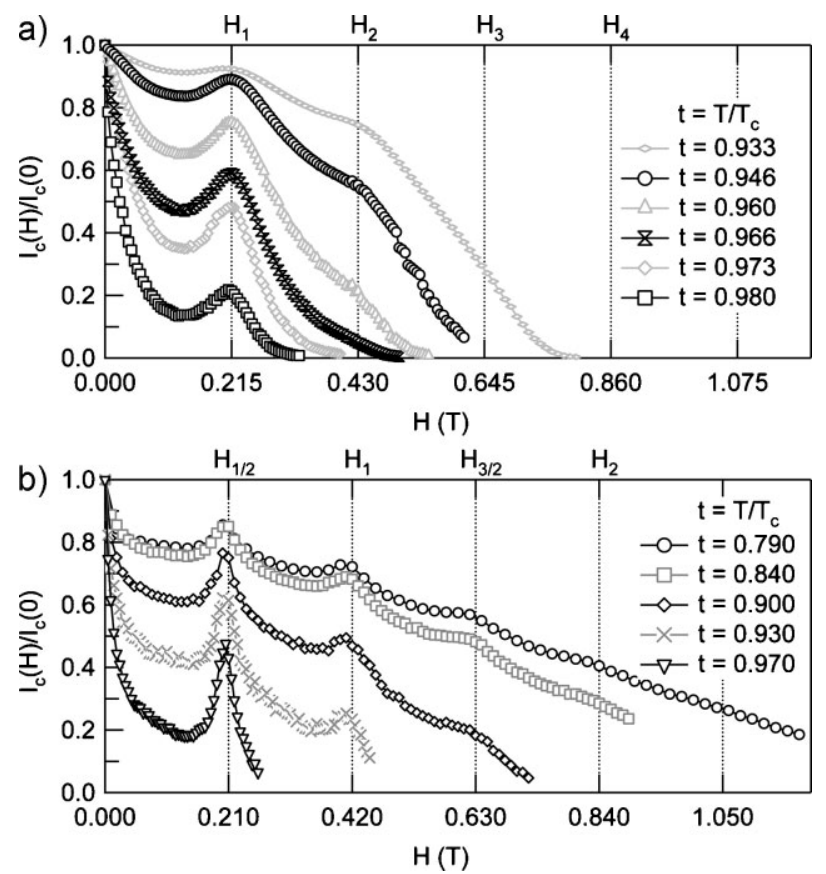

Figure 2. Normalized critical-current curves versus magnetic field for different reduced temperatures of a) a $50-\mathrm{nm} \mathrm{Nb}$ film with triangular lattice of antidots (evaporated perpendicularly onto the top surface) and b) a $50-\mathrm{nm} \mathrm{Nb}$ film with a quasi-hexagonal thickness modulation (evaporated under $30^{\circ}$ from normal onto the bottom surface). 

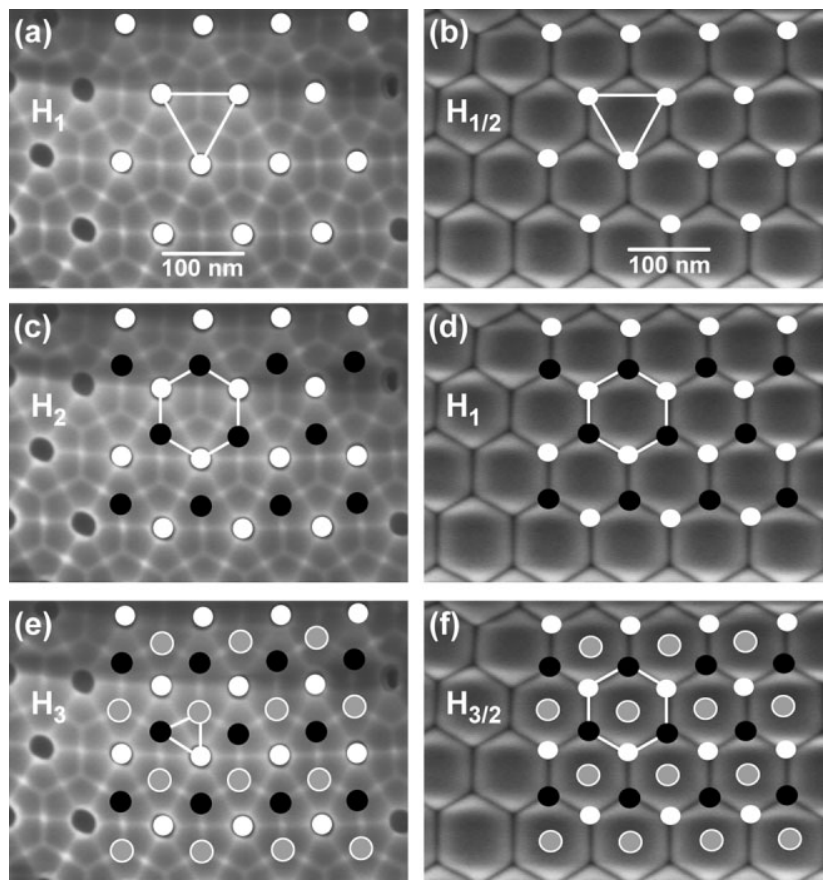

Figure 3. Vortex patterns for the 50-nm-thick $\mathrm{Nb}$ film with a triangular lattice of antidots of $\approx 100-\mathrm{nm}$ spacing at a) $H_{1}^{\text {top }}=215 \mathrm{mT}$,

c) $H_{2}^{\text {top }}=430 \mathrm{mT}$, and e) $H_{3}^{\text {top }}=645 \mathrm{mT}$, and the 50 -nm-thick sample evaporated under $30^{\circ}$ on the barrier layer surface at b) $H_{1 / 2}^{\text {bottom }}=210 \mathrm{mT}$, d) $H_{1}^{\text {bottom }}=420 \mathrm{mT}$, and f) $H_{3 / 2}^{\text {bottom }}=630 \mathrm{mT}$.

drops because of the appearance of weakly pinned interstitial vortices. At $H_{2}^{\text {top }} \approx 430 \mathrm{mT}$ a shoulder-like behavior is observed corresponding to the second matching field. The vortex lattice is then hexagonal and composed by one half of strongly pinned vortices (sitting on the holes) and one half of unpinned vortices (between the holes) (Figure 3c). Even if the effect at the third matching field is theoretically predicted to be more pronounced than at the second due to the formation of a triangular lattice of vortices ${ }^{[11]}$ (Figure 3e), it is not observed here probably because of the imperfect order of the hole lattice. As also observed in Reference ${ }^{[5]}$ for lithographically patterned samples, matching effects are usually only observed very close to the critical temperature, $T_{\mathrm{c}}$ (typically $\left.t=T / T_{\mathrm{c}}>0.95\right)$. Figure $2 \mathrm{~b}$ shows the critical-current curves at different temperatures obtained for the thickness-modulated $\mathrm{Nb}$ film deposited on the bottom surface of our template. In this case, well-pronounced matching effects are observed at 210, 420, and $630 \mathrm{mT}$. In contrast to the antidot lattice, matching effects are preserved at lower temperatures. At 210 $\mathrm{mT}\left(H_{1 / 2}^{\text {bottom }}\right)$, the sharp increase of the critical current corresponds to the filling of the triangular interstice lattice with the strong pinning centers (Figure 3b). Above $210 \mathrm{mT}$, the additional vortices are placed on the other triangular lattice with weaker pinning sites. This limits the decrease of the critical current compared to the sample with the triangular lattice of antidots. At $420 \mathrm{mT}$ (Figure 3d), all the pinning centers are occupied $\left(H_{1}^{\text {bottom }}\right)$ forming a hexagonal lattice of vortices. At higher fields, interstitial vortices appear but these vortices are caged in the hexagons formed by the pinned vortices, which allows a relatively high critical current to be maintained. At $630 \mathrm{mT}$ (Figure 3f), each hexagon contains a vortex confined in its center forming a relatively stable triangular lattice of vortices, which explains again the enhanced critical current. These results correspond quite well to theoretical results obtained for hexagonal lattices. ${ }^{[11]}$ The major interest in our technique to create pinning centers is that we obtain a quasi-hexagonal lattice of pinning centers that shows essentially the same property at $H_{1 / 2}^{\text {bottom }}$ as the lattice of antidots at $H_{1}^{\text {top }}$ but performs better at higher field because the number of pinning sites is twice as high. Moreover, the hexagonal arrangement allows caging of interstitial vortices.

In summary, we have shown that the barrier layer of highly ordered AAO membranes reveals a previously unexploited nanostructured surface that can be used as a novel template. This template consists of a triangular lattice of hemispherical nanoscale bumps. We have shown its usefulness in creating a new kind of superconducting pinning lattice. $\mathrm{Nb}$ thin films have been evaporated for that purpose onto this surface, leading to a modulation of the film thickness. The corresponding pinning potential structure can be seen as two interpenetrating triangular lattices (of different strengths) equivalent to a quasi-hexagonal one. Critical-current measurements have been compared between thin $\mathrm{Nb}$ films presenting this quasihexagonal pinning center arrangement and those presenting a triangular lattice of antidots. The major advantage in choosing the barrier layer is that it doubles the number of pinning sites and matching effects are preserved at higher fields and lower temperatures.

\section{Experimental Section}

AAO templates were fabricated by a two-step anodization process in oxalic acid $(0.3 \mathrm{~m})$ at $2{ }^{\circ} \mathrm{C}$ under a constant voltage of $40 \mathrm{~V}$ to produce a $100-\mathrm{nm}$ pore spacing. A 500- $\mu \mathrm{m}$-thick ultrapure (99.999\%) Al foil was first electropolished, then partially anodized to a depth of about $50 \mu \mathrm{m}$ to promote the ordering. This anodized layer was then selectively removed by chemical etching with a mixture of $\mathrm{H}_{3} \mathrm{PO}_{4}(6 \mathrm{wt} \%)$ and chromic acid $(1.8 \mathrm{wt} \%)$ at $60{ }^{\circ} \mathrm{C}$. A second anodization $(20 \mu \mathrm{m})$ under the same conditions was then performed in order to obtain a highly ordered structure.

To use the barrier-layer surface (at the bottom) for subsequent $\mathrm{Nb}$ deposition and to be able to manipulate the sample, the top surface of the template (presenting holes) was stuck onto a silicon wafer with commercial epoxy resin (Stycast). The remaining Al that was not anodized was selectively removed with a solution of $\mathrm{CuCl}_{2}$ $(0.125 \mathrm{M})$ and $\mathrm{HCl}(6 \mathrm{M})$, revealing the AAO surface, which shows an ordered arrangement of nanoscale bumps with a triangular lattice. $\mathrm{Nb}$ depositions were performed using MBE at room temperature at a working pressure of $10^{-10}$ torr and at an evaporation rate of 1.5 $\AA \mathrm{s}^{-1}$. The $T_{\mathrm{c}}$ obtained on $\mathrm{Si}$ as well as on the bumps and holes sides of AAO amounted to $8.9,7$, and $5.35 \mathrm{~K}$, respectively.

\section{Keywords:}

alumina - hexagonal arrangement - nanostructures . superconductivity $\cdot$ vortex pinning 


\section{communications}

[1] a) H. Masuda, K. Fukuda, Science 1995, 268, 1466-1468; b) O. Rabin, P. R. Hertz, Y.-M. Lin, A. I. Akinwande, S. B. Cronin, M. S. Dresselhaus, Adv. Funct. Mater. 2003, 13, 631-638; c) W. Lee, R. Ji, U. Gösele, K. Nielsch, Nat. Mater. 2006, 5, 741747; d) L. Piraux, K. Renard, R. Guillemet, S. Mátéfi-Tempfli, M. Mátéfi-Tempfli, V. Antohe, S. Fusil, K. Bouzehouane, V. Cros, Nano Lett. 2007, 7, 2563-2567.

[2] a) A. Vlad, M. Mátéfi-Tempfli, V. A. Antohe, S. Faniel, N. Reckinger, B. Olbrechts, A. Crahay, V. Bayot, L. Piraux, S. Melinte, S. Mátéfi-Tempfli, Small 2008, 4, 557-560; b) S. Mátéfi-Tempfli, in Leading Edge Nanotechnology Research Developments, (Ed: D. M. Sabatini), Nova Science Publishers, New York 2008, pp. 235-256.

[3] A. A Abrikosov,, Sov. Phys. JETP 1957, 5, 1174-1182.

[4] a) O. Daldini, P. Martinoli, J. L. Olsen, G. Berner, Phys. Rev. Lett. 1974, 32, 218-221; b) A. T. Fiory, A. F. Hebard, S. Somekh, Appl. Phys. Lett. 1978, 32, 73-75; c) A. Bezryadin, B. Pannetier, J. Low Temp. Phys. 1995, 98, 251-268; d) M. Baert, V. V. Metlushko, R. Jonckheere, V. V. Moshchalkov, Y. Bruynseraede, Phys. Rev. Lett. 1995, 74, 3269-3272; e) D. J. Morgan, J. B. Ketterson, Phys. Rev. Lett. 1998, 80, 3614-3617; f) T. C. Wu, J. C. Wang, L. Horng, J. C. Wu, T. J. Yang, J. Appl. Phys. 2005, 97, $10 \mathrm{~B} 102$.

[5] A. V. Silhanek, L. Van Look, R. Jonckheere, B. Y. Zhu, S. Raedts, V. V. Moshchalkov, Phys. Rev. B 2005, 72, 014507.

[6] U. Welp, Z. L. Xiao, J. S. Jiang, V. K. Vlasko-Vlasov, S. D. Bader, G. W. Crabtree, J. Liang, H. Chik, J. M. Xu, Phys. Rev. B 2002, 66, 212507.
[7] W. Vinckx, J. Vanacken, V. V. Moshchalkov, J. Appl. Phys. 2006, 100, 044307.

[8] a) V. V. Moshchalkov, L. Gielen, C. Strunk, R. Jonckheere, X. Qiu, C. Van Haesendonck, Y. Bruynseraede, Nature 1995, 373, 319322; b) V. V. Moshchalkov, M. Baert, V. V. Metlushko, E. Rosseel, M. J. VanBael, K. Temst, Y. Bruynseraede, R. Jonckheere, Phys. Rev. B 1998, 57, 3615-3622; c) W. Vinckx, J. Vanacken, V. V. Moshchalkov, S. Mátéfi-Tempfli, M. Mátéfi-Tempfli, S. Michotte, L. Piraux, X. Ye, J. Phys. C 2007, 459, 5-10; d) J. Vanacken, W. Vinckx, V. V. Moshchalkov, S. Mátéfi-Tempfli, M. Mátéfi-Tempfli, S. Michotte, L. Piraux, X. Ye, J. Phys. C 2008, 468, 585-588.

[9] W. Vinckx, J. Vanacken, V. V. Moshchalkov, S. Mátéfi-Tempfli, M. Mátéfi-Tempfli, S. Michotte, L. Piraux, Eur. Phys. J. B 2006, 53, 199-203.

[10] S. Mátéfi-Tempfli, M. Mátéfi-Tempfli, L. Piraux, Thin Solid Films 2008, 516, 3735-3740.

[11] C. Reichhardt, C. J. Olson Reichhardt, Phys. Rev. B 2007, 76, 214305.
Received: March 6, 2009 Revised: May 29, 2009

Published online: July 27, 2009 